Int. J. Dev. Biol. 51: 707-714 (2007)

doi: $10.1387 /$ ijdb.072450yj

Original Article

\title{
DNA methylation state is preserved in the sperm-derived pronucleus of the pig zygote
}

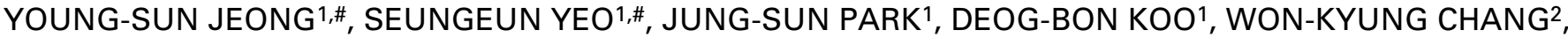 \\ KYUNG-KWANG LEE ${ }^{1}$ and YONG-KOOK KANG ${ }^{1, *}$ \\ ${ }^{1}$ Center for Regenerative Medicine, KRIBB, 52 Oun-dong, Yuseong-gu, Daejon, South Korea and \\ ${ }^{2}$ National Livestock Research Institute, Suwon, Korea.
}

\begin{abstract}
DNA methylation reprogramming (DMR) during preimplantation development erases differentiation-associated, unessential epigenetic information accumulated during gametogenesis, and ultimately brings pluripotency to the resulting embryo. Two patterns of DMR of spermderived pronucleus have been reported in mammals. In the first, the male pronucleus is actively demethylated whereas in the second, the methylation state seems to be maintained. The maintenance-type DMR has been seen only through immunocytochemical observations, and waits to be proven by additional molecular-level evidence. We demonstrate that, in pig, paternally derived DNA methylation is preserved during pronucleus development, based on the following observations. First, immunostaining of pig zygotes at different time points showed the DNA methylation state to be balanced between parental pronuclei throughout pronucleus development. Second, bisulfite analysis of $P R E-1$ repetitive sequences found mono- and polyspermic eggs to have similar methylation states. Third, the methylation state of a human erythropoietin gene delivered by transgenic pig spermatozoa was maintained in the male pronucleus. Finally, 5-aza2 '-deoxycytidine treatment, which blocks re-methylation, did not show the male pronucleus to be stalled in a demethylated state. In pig zygotes, paternally derived cytosine methylation was preserved throughout pronucleus development. These findings from multilateral DMR analyses provide further support to the view that DMR occurs in a non-conserved manner during early mammalian development.
\end{abstract}

KEY WORDS: DNA methylation, demethylation, reprogramming, pig embryos, male pronucleus

\section{Introduction}

Zygotic genome reprogramming resets unique epigenetic information of the gametes, ultimately leading the zygote to reach pluripotency (Reik etal., 2001, Rideout et al., 2001, Li, 2002). The level of DNA methylation, which is known to be a target in genome reprogramming, fluctuates markedly in mice owing to an activemode demethylation process in the male pronucleus of a fertilized oocyte and a replication-coupled, passive demethylation process between successive cleavage stages (Mayer et al., 2000, Santos et al., 2002). The dynamic change in DNA methylation, or DNA methylation reprogramming (DMR, (Park et al., 2007)), in mice was believed to be conserved in mammals, and the significance of genome-wide demethylation in the paternal genome was highlighted by the hypothesis that it plays a role in controlling imprinting in mammalian development (Oswald et al., 2000, Reik and Walter, 2001a, Reik and Walter, 2001b, Barton et al., 2001). In agreement with this, it was reported that paternally derived DNA methylation was actively lost during pronucleus development in rat, cow, and pig zygotes (Dean et al., 2001).

Subsequent studies, however, reported the contrasting observation that the DNA methylation state was maintained in the male pronucleus in zygotes of other mammals such as sheep (Beaujean et al., 2004a) and rabbit (Beaujean et al., 2004a, Shi et al., 2004).

Abbreviations used in this paper: DMR, DNA methylation reprogramming; 5MeC, 5-methylcytosine; hpf, hours post-fertilization; acH3, acetylated H3; H3-m ${ }_{3} \mathrm{~K} 9$, trimethylated lysine 9 of histone H3; SINE, short interspersed element; epo, erythropoietin; 5-azadC, 5-aza-2'-deoxycytidine; DNMT1, DNA methyltransferase 1; ICM, inner cell mass; DNMT1o, oocyte-specific DNMT1; IAP, intracisternal A-type particle; Etn, early retrotransposon; DAPI, 4',6'diamidino-2-phenylindole hydrochloride.

*Address correspondence to: Yong-Kook Kang. Center for Regenerative Medicine, KRIBB, 52 Oun-dong, Yuseong-gu, Daejon, 305-600, South Korea. Tel: +82-42-860-4427. Fax.+82-42-860-4608. e-mail: ykkang@kribb.re.kr

\# These authors contributed equally to this paper. 
These observations had the blow of the active demethylation obscure and brought dubious looks on biological significance of the early DNA methylation reprogramming in the paternal genome. Presently pressing is, prior to dealing with developmental meaning of it, the characterization of DNA methylation reprogramming pattern in diverse species, which would provide us meaningful clues and eventually help understand the role of early methylation reprogramming in mammals. Immunocytochemical evidence of DNA methylation state provided by these related studies seems to be limited and unable to resolve the conflicting observations among different mammals. Therefore, multiple approaches are required to verify the existing immunocytochemical observations on early methylation reprogramming.

Here, we reports the DNA methylation reprogramming pattern of fertilized pig oocytes. We found that, in pigs, paternally derived DNA methylation was preserved throughout pronucleus development. Our conclusion was based on several lines of evidence obtained from multilateral analyses of methylation reprogramming, as follows: 1) immunocytochemical observations of pig zygotes at different time points; 2) sequence-level analyses of the CpG methylation state of mono- and polyspermic zygotes using bisulfite mutagenesis technology; 3) analysis of the methylation profile of a human erythropoietin gene of sperm origin in pig zygotes; and finally, 4) visual inspection of the effect of 5-aza-2'deoxycytidine on the paternal methylation state. Our findings support the view that DNA methylation reprogramming occurs in a non-conserved manner during early development, and that demethylation of the paternal genome is not an obligatory requirement for early development (Beaujean et al., 2004a).

\section{Results}

\section{Preservation of paternally derived DNA methylation in pig zygote - immunocytochemical evidence}

We immunocytochemically investigated the DNA methylation states of pig zygotes using a monoclonal antibody that specifically recognizes 5-methylcytosine (5-MeC) (Mayer et al., 2000). When examined at 20 hours post-fertilization (hpf), pig zygotes produced by in vitro procedures showed similar methylation levels between the male and the female pronucleus (Fig. 1Aa-c). Similarly, a polyspermic egg containing multiple sperm-derived pronuclei in the cytoplasm had equivalent 5-MeC signals in all pronuclei (Fig. 1Ad-f). Anti-nuclear lamin A/C antibody was used for nuclear staining because it does not stain oocyte cytoplasm-embedded sperm heads, which can often confuse interpretation of DNA staining results (Prather et al., 1989, Jeong et al., 2007). These observations were in marked contrast to those reported for a mouse zygote (Fig. 1B); the sperm-derived DNA was actively demethylated while the maternally derived pronucleus remained methylated in mouse zygotes, creating an asymmetric methylation state between the parental pronuclei (Mayer et al., 2000, Oswald et al., 2000, Santos et al., 2002). Since mouse embryos at the preimplantation stage do not have nuclear lamin $\mathrm{A} / \mathrm{C}$ (Moreira et al., 2003), the parental pronuclei were stained for acetylated H3 (acH3), instead.

We then examined the DNA methylation states of pig zygotes at different time points $(6,15,20$, and $25 \mathrm{hpf})$ during pronucleus development (Fig. 2). The male pronucleus was distinguished from the female one by the weaker level of trimethylated histone H3- lysine $9\left(\mathrm{H} 3-\mathrm{m}_{3} \mathrm{~K} 9\right)$ (Jeong et al., 2007) and also by more decondensed chromatin state (see Fig. $2 b$ and e). These criteria are particularly useful for early- to mid-stage pig zygotes (usually those in 6 to $15 \mathrm{hpf}$, empirically) where the parental pronuclei are clearly different from each other in $\mathrm{H} 3-\mathrm{m}_{3} \mathrm{~K} 9$ level. On the contrary, for late pig zygotes (those at 18 25 hpf; Fig. $2 \mathrm{~h}$ and k) in which both parental pronuclei have equivalent $\mathrm{H} 3-\mathrm{m}_{3} \mathrm{~K} 9$ levels, it is for the most part difficult to decide the parental origins of the pronuclei. The other criteria to discern the parental pronuclei from each other such as the relative pronuclear size and the relative distance from the polar body, which are generally accepted in the rodent zygotes, are not always applicable to the zygotes of domestic animals (Young and Beaujean, 2004), especially to the pig zygotes.

As shown in Fig. 2, during the whole one-cell stage, the spermderived pronucleus maintained a substantial level of $5-\mathrm{MeC}$, and we were unable to detect evidence for an active demethylation event that leads to a marked reduction in, or a complete loss of,

\section{A}
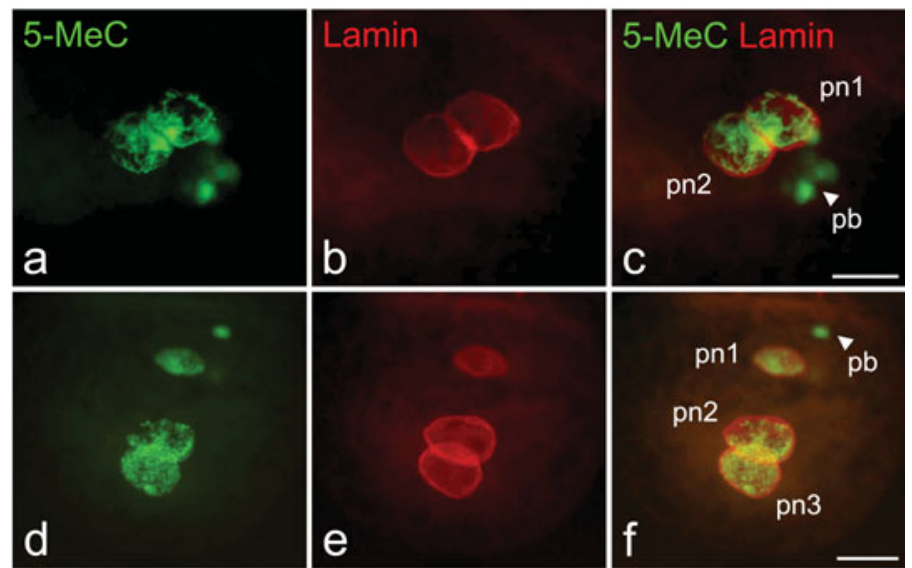

B
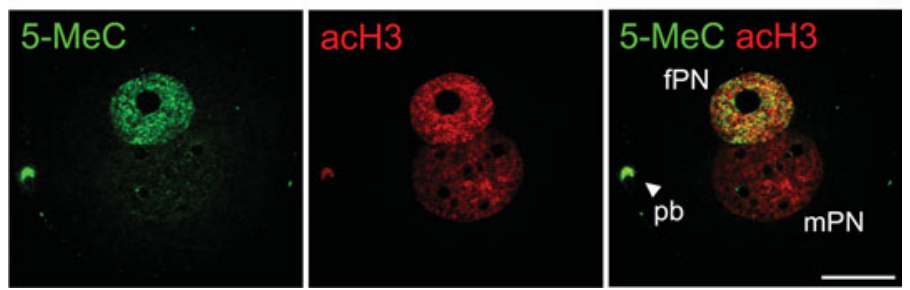

Fig. 1. Immunostaining of pig one-cell eggs for 5-methylcytosine (5MeC). (A) Monospermic (a-c) and polyspermic (d-f) pig zygotes. Pig zygotes were fixed at 20 hours post-fertilization (hpf) for staining. Note that, in a polyspermic egg $(n>100)$, all pronuclei are equivalently stained for 5-MeC. (B) Mouse zygote. 5-MeC is almost undetectable in the mouse paternal pronucleus (mPN). Samples were stained with a monoclonal antibody (Mayer et al., 2000) that specifically recognizes 5-MeC. Counterstain was done either with nuclear lamin $A / C(A)$ or acetylated $H 3$ $(\mathrm{acH} 3 ; \mathrm{B})$. Female and male pronuclei are arbitrarily designated as 'pn 1', 'pn2', etc., because, unlike in the mouse, it is difficult to assign their parents of origin. Images were captured using a Carl Zeiss Axiovert 200M microscope equipped with ApoTome, and merged using Adobe Photoshop (v7.0). fPN and mPN, paternal and maternal pronuclei; pb, polar body. Scale bar, $20 \mu \mathrm{m}$. 
cytosine methylation in the paternally derived pronucleus, as seen in the mouse zygote (Fig. 1B). Therefore, we concluded that the pig is one of the species in which the paternally derived cytosine methylation state is maintained during pronucleus development, as seen in sheep (Beaujean et al., 2004a).

\section{Sequence-level evidence for lack of active DNA demethylation in pig zygote}

Sequence-level analysis by bisulfite mutagenesis technology supported our immunostaining results. Bisulfite treatment of genomic DNA converts unmethylated, but not methylated, cytosine to uridine by a deamination reaction, which allows unmethylated and methylated cytosine bases to be distinguished in the subsequent sequencing analysis (Han et al., 2006).

The genomic methylation states of the $P R E-1$ repetitive sequences were analyzed in pig mature oocytes and spermatozoa using bisulfite sequencing technology. $P R E-1$ is a pig endogenous short interspersed element (SINE) that is present at high copy number throughout the euchromatin regions of the pig genome (Kang et al., 2001b). As shown in Fig. 3A, 42\% of PRE-1 sequences were methylated in the mature oocyte (of $97 \mathrm{CpG}$ sites examined, $41 \mathrm{CpGs}$ were methylated) and 53\% methylated in the spermatozoa (69 of $130 \mathrm{CpGs}$ examined). For methylation analysis of fertilized zygotes, we first divided the zygotes into two subgroups according to the number of pronuclei observed using a light microscope equipped with a micromanipulator system: 1) a monospermic group that contained two pronuclei (one from the male and one from the female); and 2) a polyspermic group that contained more than four pronuclei (one from the female and the rest from the male). The rationale for this experiment is that, if active demethylation occurs throughout the genome resulting in loss of 5-MeCs from the sperm-derived pronucleus, the polyspermic group would have a greatly reduced level of genomic methylation compared with the monospermic group. However, bisulfite sequencing analysis revealed that the monospermic and polyspermic zygotes were 56\% (57/102) and 46\% (58/127) methylated, respectively. The similar methylation rates between the two groups indicate that the paternal copies of $P R E-1$ sequences are not demethylated during the pronucleus stage.

Analysis of a transgenic sequence that is present only in the sperm-derived genome gave consistent results. The use of transgenic sperm enabled us to monitor methylation changes at unique sequences of male origin. Bisulfite sequencing analysis of transgenic pig spermatozoa containing the human erythropoietin (epo) gene found that this human eposequence was heavily methylated (Fig. 3B). The DNA methylation states of pig oocytes infiltrated by the transgenic spermatozoa were examined. At 20-22 hpf, when the first zygotic DNA replication is almost complete according to our BrdU incorporation analysis (Jeong et al., 2007), most of the CpGs, except the first and the third CpG sites, remained methylated. Although we observed point demethylation at the epo sequence (see discussion), the results support a lack of global demethylation on sperm-derived DNA during the pronucleus stage in pig zygotes.

\section{Paternal level of DNA methylation is not affected by treat- ment with 5-aza-2'-deoxycytidine}

The apparent retention of the gametic DNA methylation pattern in the male pronucleus could be due to the lack of active

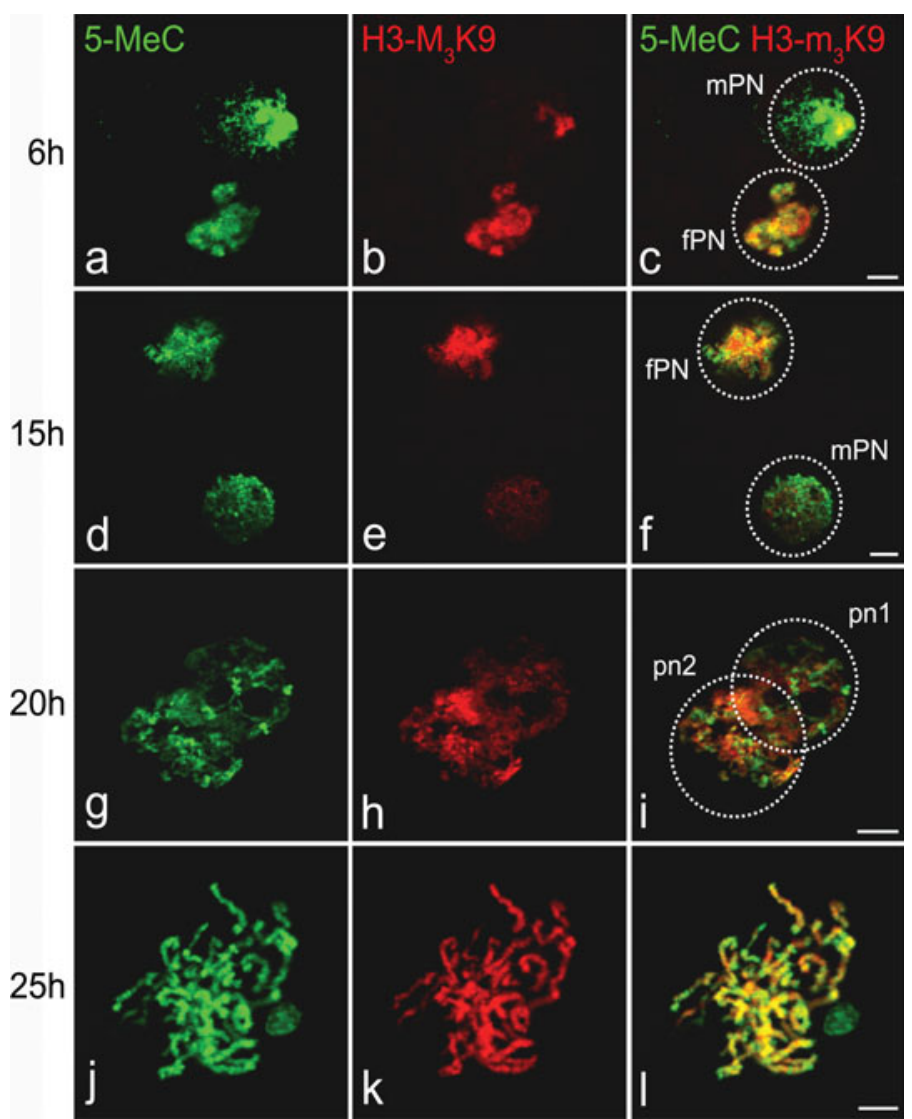

Fig. 2. DNA methylation state of paternal genome is preserved throughout the pronucleus stage in pig. Fertilized pig oocytes were collected at different time points $(6,15,20$ and 25 hours post-fertilization [hpf]) and fixed for staining for 5-MeC and trimethylated lysine 9 of histone $\mathrm{H} 3\left(\mathrm{H} 3-m_{3} \mathrm{~K} 9\right)$. Note that the paternal pronucleus at given time points shows an unchanged DNA methylation state and no genomewide loss of 5-MeC. Of the two parental pronuclei, the one that shows the lower (at 6 and $15 \mathrm{hpf}$ ) $\mathrm{H} 3-\mathrm{m}_{3} \mathrm{~K} 9$ signal is identified as the paternally derived pronucleus. At $25 \mathrm{hpf}$, when condensed chromosome fibers are shown, the two parental pronuclei show the same level of $\mathrm{H}_{3}-\mathrm{m}_{3} \mathrm{Kg}$. Boundaries of pronuclei are indicated by dashed circles in the merged images. For pn 1, pn2, mPN, andfPN, see the legend of Fig. 1. Images were captured using a Carl Zeiss Axiovert 200M microscope equipped with ApoTome, and merged using Adobe Photoshop (v7.0). Scale bar, $10 \mu \mathrm{m}$.

demethylation or to the effects of active demethylation and de novo DNA methylation processes that take place simultaneously in the paternal pronucleus. To determine which is the case, pig zygotes were treated with 5-aza-2'-deoxycytidine (5-azadC) to block de novo DNA methylation processes by inhibiting DNA methyltransferase I (DNMT1) and, possibly, DNMT3A and DNMT3B (Christman, 2002). 5-azadC does not inhibit the demethylation process; thus if an active demethylation event occurred in the pig male pronucleus, 5-azadC treatment would show the paternal pronucleus to be demethylated.

Fertilized pig oocytes were treated with 5-azadC at various concentrations $(5,50$ and $500 \mu \mathrm{M})$ and double-stained for $5-\mathrm{MeC}$ and $\mathrm{H} 3-\mathrm{m}_{3} \mathrm{~K} 9$ (Fig. 4). As shown by staining for $5-\mathrm{MeC}$, the methylation levels of the parental pronuclei were very similar, with no sign of global loss of DNA methylation in the male pronucleus. 
As a control, we confirmed the demethylation effect of 5-azadC even at a concentration of $5 \mu \mathrm{M}$ in bovine zygotes (Park et al., 2007). This result further verifies the lack of an active DNA demethylation event in the pig paternal pronucleus.

\section{Methylation level of zygotic genome seems to be maintained without alteration between cleavage stages - lack of passive demethylation in early pig development}

We then analyzed cleavage-stage pig embryos for evidence of a replication-coupled, passive-mode DNA demethylation event known to occur in mice (Rougier et al., 1998). Immunostaining of preimplantation-stage pig embryos for $5-\mathrm{MeC}$ showed that there

A

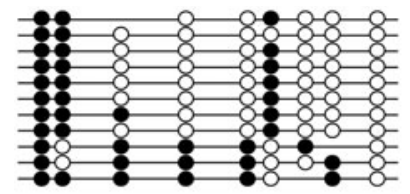

Oocyte $42 \%(41 / 97)$

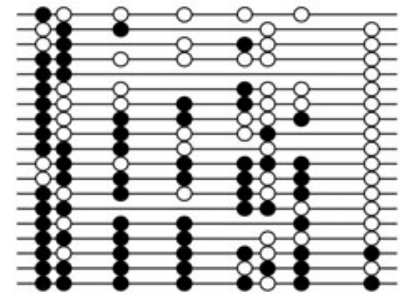

Sperm 53\% (69/130)

B

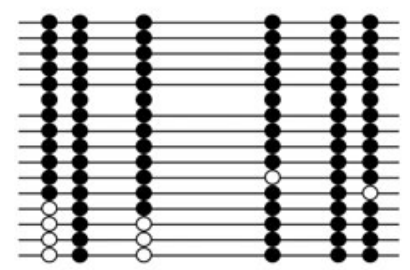

Sperm (epo)

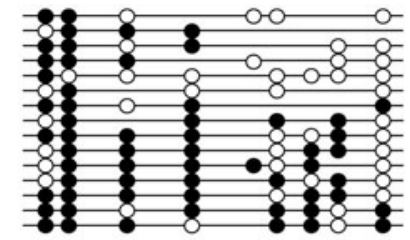

Mono-sp 56\% (57/102)

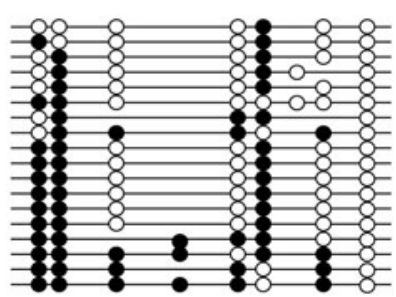

Poly-sp 46\% (58/127)

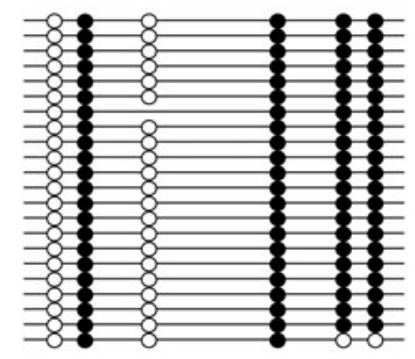

One-cell (epo) was no discernible alteration in the DNA methylation level between successive cleavage stages (Fig. 5, left panels), indicating that passive demethylation does not occur at the pre-implantation stage. For a positive reference, we also examined cleavagestage mouse embryos simultaneously for DNA methylation states (Fig. 5, right panels); a gradual decrease of 5-MeC signal was evident between the stages, though the inner-cell mass (ICM)specific re-methylation was not detected in the mouse blastocysts (Santos et al., 2002). These observations suggest that the DNA methylation level of the zygotic genome is maintained by a process coupled to DNA replication, like that of the somatic genome (Bestor, 2000). Taken all together, our results indicate that genome-wide DNA demethylation processes such as activeand passive-mode demethylation do not occur in pig early development.
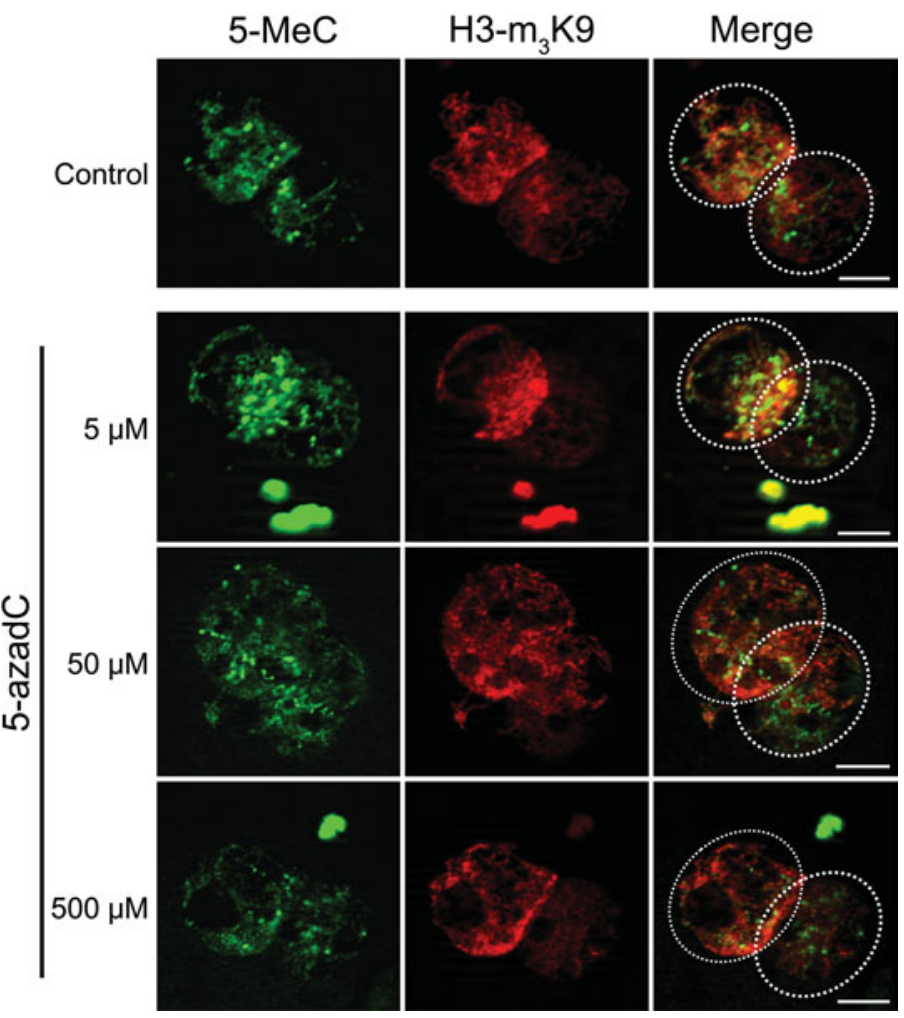

Fig. 3 (Left). CpG methylation profiles of pig genomic sequences. (A) PRE-1 repetitive sequences. A 148-bp CpG-rich region of pig PRE-1 short interspersed element (SINE) was analyzed using bisulfite mutagenesis technology in the mature oocytes, spermatozoa, and fertilized oocytes. PRE1 showed a varied $C p G$ distribution between PCR strings, which is a typical pattern of repetitive sequences of high copy number that are widely dispersed throughout the pig genome. Polyspermic eggs $(n>150)$ with four pronuclei were selected under a light microscope (200x) equipped with a micromanipulation system (Leica). (B) Methylation profile of paternally derived human erythropoietin (epo) gene sequence in transgenic pig zygotes. A 214-bp sequence of the human erythropoietin gene (GenBank\# M11319.1) was analyzed by bisulfite sequencing. Methylation states of the human epo sequence in transgenic spermatozoa (Sperm) and in the transgenic pig zygotes (One-cell) are shown. Samples were collected at 20-22 hpf for preparation of genomic DNA. Blank and filled circles indicate unmethylated and methylated CpGs, respectively. The methylation percentage is the proportion of all CPG sites examined that were methylated. Mono-sp and Poly-sp, fertilized oocytes infiltrated by single or multiple sperm, respectively.

Fig. 4 (Right). Preservation of DNA methylation in the male pronucleus treated with 5-aza-2'-deoxycytidine. In-vitro-fertilized pig oocytes were incubated in the medium containing DMSO only (Control) or given concentrations of 5-aza-2'-deoxycytidine (5-azadC) for about 20 hours. Pig zygotes were double-stained for 5-MeC (gray in 5-MeC panels and green in merged panels) and H3- $m_{3} \mathrm{~K} 9$ (red). Note that both parental pronuclei have similar levels of DNA methylation, regardless of 5-azadC treatment. Boundaries of parental pronuclei are indicated by dashed circles. Scale bar, $10 \mu \mathrm{m}$. 

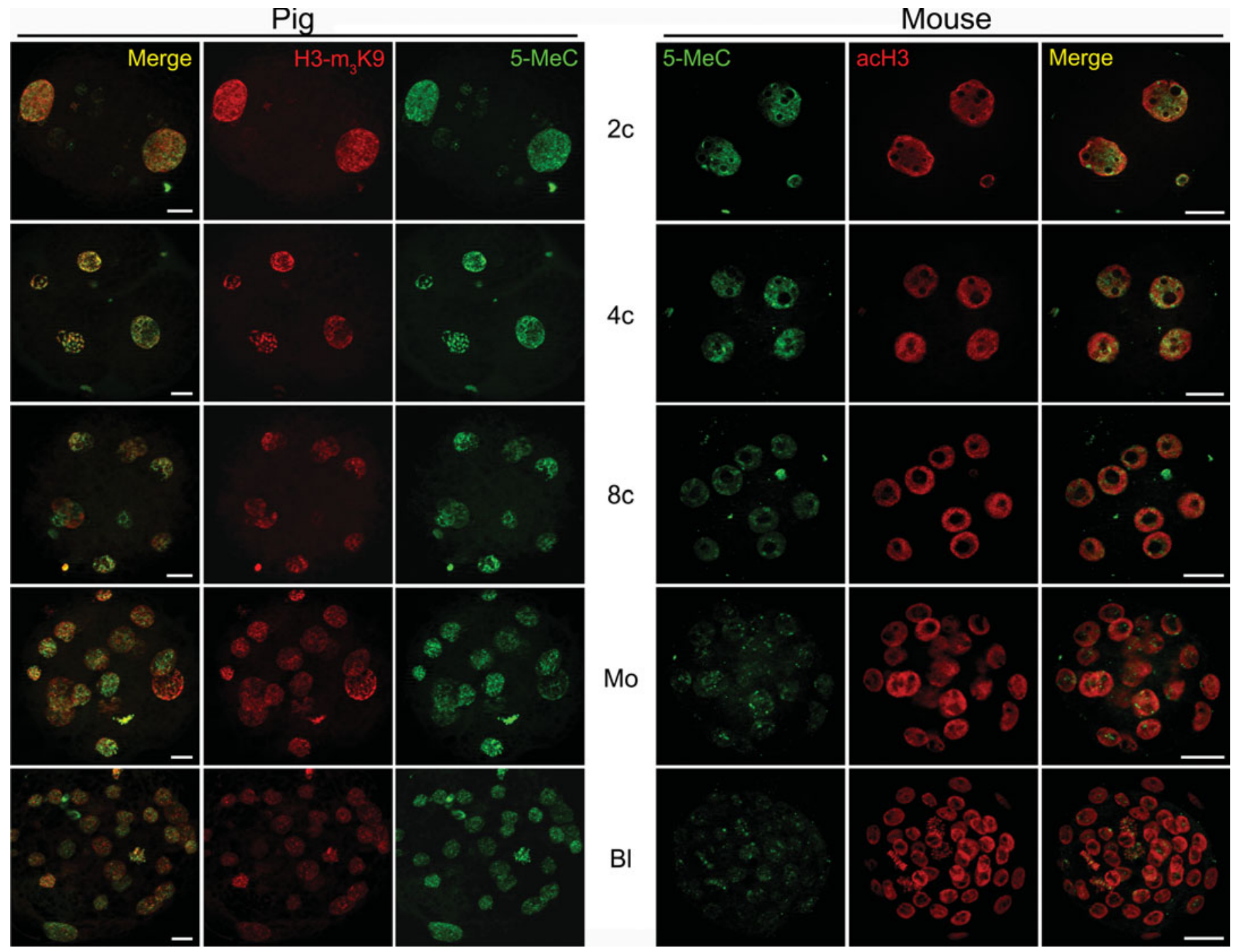

Fig. 5. Maintenance of DNA methylation state during cleavage stages in pig. (Left) Pig embryos. Approximately 50 embryos at each cleavage stage were collected and stained for 5-MeC. There is no detectable loss of 5-MeC level between successive stages. (Right) Mouse embryos. Twenty embryos per each stage were used for 5-MeC staining. Counterstain was done with either H3-m $\mathrm{K}_{3}$ (pig) or acetylated H3 (acH3; mouse). Unlike the mouse embryos, the nuclei of which clearly show a figure of gradual demethylation between the successive stages, the pig embryos outwardly maintain their genomic DNA methylation levels throughout the preimplantation stage. $2 \mathrm{c}, 4 \mathrm{c}$ and $8 \mathrm{c}$ indicate two-, four- and eight-cell embryos, respectively. Mo, morula; Bl, blastocyst. Images were captured using a Carl Zeiss Axiovert 200M microscope equipped with ApoTome, and merged using Adobe Photoshop (v7.0). Scale bar, $20 \mu \mathrm{m}$.

\section{Discussion}

The results of bisulfite mutagenesis (Fig. 3) are, overall, consistent with the immunocytochemical observations (Fig. 1 and 2) and support the lack of an active demethylation event in the pig male pronucleus. We obtained methylation profiles from one-cell eggs at 20-22 hpf when the DNA replication cycle is almost complete (Jeong et al., 2007) and chromatins become condensed to prepare for the first mitosis in pig zygotes. Therefore, the $\mathrm{CpG}$ methylation profiles of paternally derived human epo transgene sequence provide useful information about pig methylation reprogramming processes (Fig. 4). First, the homogeneous CpG methylation pattern among different PCR clones indicates that sperm- derived CpG methylation patterns are faithfully inherited by daughter DNA strands during the first round of DNA replication, possibly by the same mechanism by which somatic cells maintain DNA methylation levels (Bestor, 2000). Second, we observed some demethylation at certain $\mathrm{CpG}$ dinucleotides in the transgene sequence, so the site-specific loss of $\mathrm{CpG}$ methylation indicates the existence of a DNA demethylation activity in pig fertilized oocytes. The demethylation activity was confined to certain $\mathrm{CpG}$ sites without spreading into nearby $\mathrm{CpG}$ sites, so this 'point' demethylation is likely to result from a mechanism (such as demethylation by DNA binding proteins) that is distinct from the 'global' and 'active' demethylation mechanism that occurs in the mouse fertilized oocyte. 
The species-dependence of the passive-mode demethylation process might be simply attributed to the cellular localization of functional DNMT1 (Bestor, 2000). Immunostaining results have shown that the oocyte-specific DNMT1 (DNMT10) with intact DNA methylating activity is present in pre-implantation-stage mouse embryos but is excluded from the embryonic nucleus until the eight-cell stage (Cardoso and Leonhardt, 1999), leading to passive DNA demethylation. However, it is not known whether pig and sheep embryos contain a functional DNMT1 in their embryonic nuclei to maintain the genomic DNA methylation pattern during cleavages. We attempted to resolve this issue using several anti-DNMT1 antibodies and $d n m t 1$-overexpression constructs used for microinjection, but failed to reach any conclusion for DNMT1 localization in fertilized mammalian oocytes, probably owing to low accessibility of the antibodies used and to translational inhibition of the dnmt1 mRNA (Ratnam et al., 2002). Therefore, it is still unknown if cleavage-stage pig embryos express a functional maintenance DNA methyltransferase.

As to the maintenance of DNA methylation between the cleavage stages in pig, our immunocytochemical observation in Fig. 5 looks as if it conflicts with the previous result of passive DNA methylation in PRE-1 sequences (Kang et al., 2001b). Immunocytochemical observation of the maintenance of DNA methylation state does not mean that all the genomic regions preserve their methylation states throughout the preimplantation development; rather it implies the sum of diverse changes of DNA methylation such as maintenance methylation, demethylation and de novo methylation at different patches of genomic regions. In fact, different genomic sequences do not always behave the same. In mice, long interspersed element (LINE) repeats were demethylated during successive cleavage stages while intracisternal A-type particle (IAP) and early retrotransposon (Etn) sequences were somewhat resistant to demethylation during preimplantation development (Walsh etal., 1998, Lane et al., 2003, Kim et al., 2004). In bovine, a maintenance methylation was observed in the satellite I sequences, a decrease in the alphoid satellite, and an increase in the satellite II regions (Kang et al., 2005). Therefore, it seems common that while most of the genomic sequences tend to obey genome-wide trend, certain repeats do not follow the global direction of DNA methylation change. In this light, unfortunately, the PRE-1 sequences we analyzed before (Kang et al., $2001 b)$ may not be relevant sequences that reflect the genomewide pattern of DNA methylation change occurring in early pig development.

Our observation that the pig sperm-derived genome does not undergo active demethylation does not agree with a previous report (Dean et al., 2001) that described 'an almost complete' loss of paternally derived 5-MeCs in pig zygotes. It is unclear why there is such a contradiction between studies that used the same anti5-MeC monoclonal antibody. However, in addition to the immunocytochemical analysis, our conclusion is further supported by several lines of evidence from multilateral approaches: 1) no molecular-level difference in CpG methylation states between mono- and polyspermic zygotes (Fig. 3B);2) maintenance of CpG methylation of the human epotransgene (Fig. 4C); and 3) failure to induce a demethylated state in the male pronucleus by treatment with concentrated 5-azadC (Fig. 5). Therefore, we conclude that the paternally derived cytosine methylation is preserved during pronucleus development in pigs.
Two recent studies have also proposed a demethylation event in the pig male pronucleus (Gioia et al., 2005, Fulka et al., 2006). However, these studies differ in their interpretation of the active demethylation event. Although the two studies claimed the occurrence of active demethylation in pig zygotes, the extent of paternal DNA demethylation observed or quantified was only partial and was not a genome-wide process. Therefore, the results of these two studies disagree with the previous report describing comprehensive demethylation in the pig zygote (Dean et al., 2001). We believe that quantification of signal intensity between paternal and maternal pronuclei is an important consideration. Two parental pronuclei are, in general, different in size and in stage, and contain chromatins with different degrees of condensation. These factors can all interfere with exact quantification of a paternal pronucleus signal relative to a maternal one. Also, it can very often be difficult to determine the parent of origin of the pronucleus,particularly of the early-stage pronucleus, because the criteria are not strict. We overcame the difficulties in determining gender and staging the pig pronuclei by exploiting differential $\mathrm{H} 3-\mathrm{m}_{3} \mathrm{~K} 9$ staining properties of parental pronuclei: the earlier stage male pronucleus is not or only weakly stained compared with the female counterpart (Fig. 2). If 'active demethylation' could be defined as a comprehensive loss of 5-MeCs that occurs throughout the genome independently of DNA replication process, presumably by an enzymatic process as was first observed from the mouse zygote, we should be cautious when interpreting the observations described above as due to an 'active' demethylation event.

In this study, we demonstrated that the genome of an early pig embryo is not subject to active demethylation at the pronucleus stage and not to passive demethylation during the cleavage stages. The distinct DNA methylation characteristics of the pig zygote may be a mammalian epigenetic reprogramming model that is antipodal to the mouse model. Our findings support the view that DNA methylation reprogramming occurs in a nonconserved manner during early development, and that demethylation of the paternal genome is not an obligatory requirement for early development.

\section{Materials and Methods}

\section{Production of pig and mouse embryos}

Experiments were conducted according to The Animal Care and Use Committee guidelines of National Livestock Research Institute, Korea. The in vitrofertilization of matured pig oocyte was performed as reported previously (Koo etal., 2000, Koo etal., 2001). Especially, polyspermy was induced by using spermatozoa up to a concentration of $1 \times 10^{6} \mathrm{sperm} / \mathrm{ml}$. Oocytes were incubated with spermatozoa for 6 hours at $39^{\circ} \mathrm{C}$ in an atmosphere of $5 \% \mathrm{CO}_{2}$ in air. For recovery of in vivo-derived embryos, as reported previously (Kang et al., 2001b), prepubertal cyclic Landrace gilts were used. They were fed $20 \mathrm{mg}$ of altrenogest daily for 9 days beginning on Day 16 of the estrus cycle, administrated with PMSG (1500 IU, Folligon) at 24 to 30 hours after the last feeding of altrenogest and with hCG (750 IU, Pregnyl) after an additional 78 hours. The gilts were handmated or artificially inseminated 12 and 24 hours after hCG administration. Embryos were surgically collected with $50 \mathrm{ml}$ PBS from uteri by retrograde flushing.

Fertilized mouse egg was obtained as described before (Yeo et al., 2005). Briefly, embryos were derived from a cross of BCF1 (C57BL/6 x $\mathrm{CBA} / \mathrm{CA}$ ) females to BCF1 males, and were removed at an appropriate time according to the standard procedure (Hogan, 1994). Timing post- 
fertilization was judged by nuclear morphology and relative distance between parental pronuclei (Hogan, 1994).

\section{Antibodies and immunostaining}

A monoclonal anti-5-MeC antibody was purchased from Eurogentech (MMS-900S-B). Antibodies that specifically recognize trimethylated H3$\mathrm{K} 9$ residues were purchased from Upstate biotechnologies. Anti-mouse or anti-rabbit goat secondary antibodies (Alexa-488/594-conjugated form, Molecular Probes) were used to visualize the individual antigens. Immunostaining procedure appeared elsewhere (Beaujean et al., 2004b). Briefly, embryos were fixed in 4\% formaldehyde in PBS for 30 minutes at $4^{\circ} \mathrm{C}$, followed by three 20 minutes washes in $0.2 \%$ Tween 20 in PBS and permeabilized by $0.25 \%$ Triton X-100 in PBS for one hour at room temperature. The embryos were treated with $4 \mathrm{~N} \mathrm{HCl}$ for 30 minutes at room temperature and blocked for one hour at room temperature in $2 \%$ BSA, $0.1 \%$ Tween 20 in PBS. Primary antibody incubations (diluted by $1: 50-500$ ) were carried out in the blocking solution for one hour at $37^{\circ} \mathrm{C}$, followed by several washes in $0.2 \%$ Tween 20 in PBS. Secondary antibodies (diluted by 1:300-500) were incubated for 30 minutes at room temperature, followed by several washes. Embryos were mounted on Poly-prep slides (Sigma) and, after dry at room temperature, observed with Olympus epifluorescence microscope or Karl Zeiss Axiovert 200M fluorescence microscope. Most staining experiments were repeated at least three times with 20 or more embryos. Images were captured digitally using different filter sets and merged using Adobe Photoshop software (v7.0).

\section{Bisulfite treatment, PCR and sequencing}

The procedure for bisulfite mutagenesis was described elsewhere (Kang et al., 2001a). Briefly, genomic DNA of denuded pig oocytes was first digested with $B a m H I$, denatured with $0.3 \mathrm{~N} \mathrm{NaOH}$ and treated with sodium bisulfite ( $\mathrm{pH} 5$; Sigma). After the desalting and desulfonating steps, we precipitated the DNA and resuspended it in $20 \mu \mathrm{l}$ of deionized water. To amplify the target region, we did PCR twice to three times, each time using $2 \mu \mathrm{l}$ of the bisulfite-converted DNA and pooled the PCR products before cloning. On average, we used 150-300 oocytes per sample as templates for PCR. Primer sequences and PCR condition used to amplify part of the PRE-1sequence were described before (Kang et al., $2001 \mathrm{~b})$. In order to get the methylation profile of human epotransgene from transgenic sperm and fertilized pig oocytes, we amplified a $218 \mathrm{bp}$ of epogene sequence (GenBank\# M11319.1) using 40 cycles of $94^{\circ} \mathrm{C}$ for $30 \mathrm{~s}, 53^{\circ} \mathrm{C}$ for $30 \mathrm{~s}$ and $72^{\circ} \mathrm{C}$ for $30 \mathrm{~s}$. The primers used for amplifying the epo region were 5 2-CRACCTCAACTACTCCACTCC-3 2 and 5 2GTGTTTATGGGATAGGTTGG-3 2. To sequence the amplified DNA fragments, we cloned and sequenced individual $P C R$ clones using $A B I$ PRISM-377.

\section{5-aza-2'-deoxycytidine treatment of pig zygotes}

For treatment of fertilized pig oocytes with 5-aza-2'-deoxycytidine (5azadC; Sigma), pig oocytes-spermatozoa complexes were transferred to IVF medium (Koo et al., 2000) containing either five-, 50- or 500- $\mu \mathrm{M}$ 5azadC three hours after the commencement of in vitro fertilization, and cultured for 3 hours. The complexes were then transferred to 5-azadCcontaining IVC medium (Koo et al., 2000) and further cultured for 18 - 20 hours. The fertilized embryos were fixed for immunostaining in $4 \%$ formaldehyde solution (Sigma Aldrich) for 30 minutes at room temperature.

\section{Acknowledgements \\ This work was supported by KRCF/KRIBB (KGM1310713), MOST (F104AD010004) and RDA (Bio-Green 21, 2007040134017).}

\section{References}

BARTON, S.C., ARNEY, K.L., SHI, W., NIVELEAU, A., FUNDELE, R., SURANI,
M.A. and HAAF, T. (2001). Genome-wide methylation patterns in normal and uniparental early mouse embryos. Hum Mol Genet 10: 2983-7.

BEAUJEAN, N., HARTSHORNE, G., CAVILLA, J., TAYLOR, J., GARDNER, J., WILMUT, I., MEEHAN, R. and YOUNG, L. (2004a). Non-conservation of mammalian preimplantation methylation dynamics. Curr Bio/14: R266-7.

BEAUJEAN, N., TAYLOR, J.E., MCGARRY, M., GARDNER, J.O., WILMUT, I., LOI, P., PTAK, G., GALLI, C., LAZZARI, G., BIRD, A. et al. (2004b). The effect of interspecific oocytes on demethylation of sperm DNA. Proc Nat/ Acad Sci USA 101: 7636-40.

BESTOR, T.H. (2000). The DNA methyltransferases of mammals. Hum Mol Genet 9: 2395-402.

CARDOSO, M.C. and LEONHARDT, H. (1999). DNA methyltransferase is actively retained in the cytoplasm during early development. J Cel/ Bio/147: 25-32.

CHRISTMAN, J.K. (2002). 5-Azacytidine and 5-aza-2'-deoxycytidine as inhibitors of DNA methylation: mechanistic studies and their implications for cancer therapy. Oncogene 21: 5483-95.

DEAN, W., SANTOS, F., STOJKOVIC, M., ZAKHARTCHENKO, V., WALTER, J., WOLF, E. and REIK, W. (2001). Conservation of methylation reprogramming in mammalian development: aberrant reprogramming in cloned embryos. Proc Natl Acad Sci USA 98: 13734-8.

FULKA, J., FULKA, H., SLAVIK, T., OKADA, K. and FULKA, J., JR. (2006). DNA methylation pattern in pig in vivo produced embryos. Histochem Cel/ Bio/126: 213-7.

GIOIA, L., BARBONI, B., TURRIANI, M., CAPACCHIETTI, G., PISTILLI, M.G., BERARDINELLI, P. and MATTIOLI, M. (2005). The capability of reprogramming the male chromatin after fertilization is dependent on the quality of oocyte maturation. Reproduction 130: 29-39.

HAN, Y.M., KIM, S.H. and KANG, Y.K. (2006). Analysis of DNA methylation profiles in preimplantation embryos using bisulfite mutagenesis. Methods Mol Bio/325: 251-60.

HOGAN, B. (1994). Manipulating the mouse embryo: a laboratory manual. Cold Spring Harbor Laboratory Press, Plainview, N.Y.

JEONG, Y.S., YEO, S., PARK, J.S., LEE, K.K. and KANG, Y.K. (2007). Gradual development of a genome-wide H3-K9 trimethylation pattern in paternally derived pig pronucleus. Dev Dyn 236: 1509-16.

KANG, Y.K., KOO, D.B., PARK, J.S., CHOI, Y.H., CHUNG, A.S., LEE, K.K. and HAN, Y.M. (2001a). Aberrant methylation of donor genome in cloned bovine embryos. Nat Genet 28: 173-7.

KANG, Y.K., KOO, D.B., PARK, J.S., CHOI, Y.H., KIM, H.N., CHANG, W.K., LEE, K.K. and HAN, Y.M. (2001b). Typical demethylation events in cloned pig embryos. Clues on species- specific differences in epigenetic reprogramming of cloned donor genome. J Biol Chem 27: 27.

KANG, Y.K., LEE, H.J., SHIM, J.J., YEO, S., KIM, S.H., KOO, D.B., LEE, K.K., BEYHAN, Z., FIRST, N.L. and HAN, Y.M. (2005). Varied patterns of DNA methylation change between different satellite regions in bovine preimplantation development. Mol Reprod Dev 71: 29-35.

KIM, S.H., KANG, Y.K., KOO, D.B., KANG, M.J., MOON, S.J., LEE, K.K. and HAN, Y.M. (2004). Differential DNA methylation reprogramming of various repetitive sequences in mouse preimplantation embryos. Biochem Biophys Res Commun 324: 58-63.

KOO, D.B., KANG, Y.K., CHOI, Y.H., PARK, J.S., HAN, S.K., PARK, I.Y., KIM, S.U., LEE, K.K., SON, D.S., CHANG, W.K. et al. (2000). In vitro development of reconstructed porcine oocytes after somatic cell nuclear transfer. Biol Reprod 63: 986-92.

KOO, D.B., KANG, Y.K., CHOI, Y.H., PARK, J.S., KIM, H.N., KIM, T., LEE, K.K. and HAN, Y.M. (2001). Developmental potential and transgene expression of porcine nuclear transfer embryos using somatic cells. Mol Reprod Dev 58: 1521.

LANE, N., DEAN, W., ERHARDT, S., HAJKOVA, P., SURANI, A., WALTER, J. and REIK, W. (2003). Resistance of IAPs to methylation reprogramming may provide a mechanism for epigenetic inheritance in the mouse. Genesis 35: 8893.

LI, E. (2002). Chromatin modification and epigenetic reprogramming in mammalian development. Nat Rev Genet 3: 662-73.

MAYER, W., NIVELEAU, A., WALTER, J., FUNDELE, R. and HAAF, T. (2000). Demethylation of the zygotic paternal genome. Nature 403: 501-2. 
MOREIRA, P.N., ROBL, J.M. and COLLAS, P. (2003). Architectural defects in pronuclei of mouse nuclear transplant embryos. J Cel/ Sci116: 3713-20.

OSWALD, J., ENGEMANN, S., LANE, N., MAYER, W., OLEK, A., FUNDELE, R., DEAN, W., REIK, W. and WALTER, J. (2000). Active demethylation of the paternal genome in the mouse zygote. Curr Bio/10: 475-8.

PARK, J.S., JEONG, Y.S., SHIN, S.T., LEE, K.K. and KANG, Y.K. (2007). Dynamic DNA methylation reprogramming: Active demethylation and immediate remethylation in the male pronucleus of bovine zygotes. Dev Dyn 236: 25232533.

PRATHER, R.S., SIMS, M.M., MAUL, G.G., FIRST, N.L. and SCHATTEN, G. (1989). Nuclear lamin antigens are developmentally regulated during porcine and bovine embryogenesis. Biol Reprod 41: 123-32.

RATNAM, S., MERTINEIT, C., DING, F., HOWELL, C.Y., CLARKE, H.J., BESTOR, T.H., CHAILLET, J.R. and TRASLER, J.M. (2002). Dynamics of Dnmt1 methyltransferase expression and intracellular localization during oogenesis and preimplantation development. Dev Bio/245: 304-14.

REIK, W., DEAN, W. and WALTER, J. (2001). Epigenetic reprogramming in mammalian development. Science 293: 1089-93.

REIK, W. and WALTER, J. (2001a). Evolution of imprinting mechanisms: the battle of the sexes begins in the zygote. Nat Genet 27: 255-6.

REIK, W. and WALTER, J. (2001b). Genomic imprinting: parental influence on the genome. Nat Rev Genet2: 21-32.

RIDEOUT, W.M., 3RD, EGGAN, K. and JAENISCH, R. (2001). Nuclear cloning and epigenetic reprogramming of the genome. Science 293: 1093-8.

ROUGIER, N., BOURC'HIS, D., GOMES, D.M., NIVELEAU, A., PLACHOT, M., PALDI, A. and VIEGAS-PEQUIGNOT, E. (1998). Chromosome methylation patterns during mammalian preimplantation development. Genes Dev 12: 2108-13.

SANTOS, F., HENDRICH, B., REIK, W. and DEAN, W. (2002). Dynamic reprogramming of DNA methylation in the early mouse embryo. Dev Bio/241: 172-82.

SHI, W., DIRIM, F., WOLF, E., ZAKHARTCHENKO, V. and HAAF, T. (2004). Methylation reprogramming and chromosomal aneuploidy in in vivo fertilized and cloned rabbit preimplantation embryos. Biol Reprod 71: 340-7.

WALSH, C.P., CHAILLET, J.R. and BESTOR, T.H. (1998). Transcription of IAP endogenous retroviruses is constrained by cytosine methylation. Nat Genet20: 116-7.

YEO, S., LEE, K.K., HAN, Y.M. and KANG, Y.K. (2005). Methylation changes of lysine 9 of histone $\mathrm{H} 3$ during preimplantation mouse development. Mo/ Cel/s20: 423-8.

YOUNG, L.E. and BEAUJEAN, N. (2004). DNA methylation in the preimplantation embryo: the differing stories of the mouse and sheep. Anim Reprod Sci82-83: $61-78$

Edited by: Makoto Asashima Published Online: 1st October 2007

\section{Related, previously published Int. J. Dev. Biol. articles}

See our Special Issue Mammalian Reproduction \& Development edited by Brigid Hogan at: http://www.ijdb.ehu.es/web/contents.php?vol=45\&issue $=3$

Dynamic distribution of the replacement histone variant $\mathrm{H} 3.3$ in the mouse oocyte and preimplantation embryos

Maria-Elena Torres-Padilla, Andrew J. Bannister, Paul J. Hurd, Tony Kouzarides and Magdalena Zernicka-Goetz

Int. J. Dev. Biol. (2006) 50: 455-461

Histone methylation defines epigenetic asymmetry in the mouse zygote. Katharine L Arney, Siqin Bao, Andrew J Bannister, Tony Kouzarides and M Azim Surani Int. J. Dev. Biol. (2002) 46: 317-320

Epigenetic reprogramming of the genome-from the germ line to the embryo and back again. K L Arney, S Erhardt, R A Drewell and M A Surani Int. J. Dev. Biol. (2001) 45: 533-540

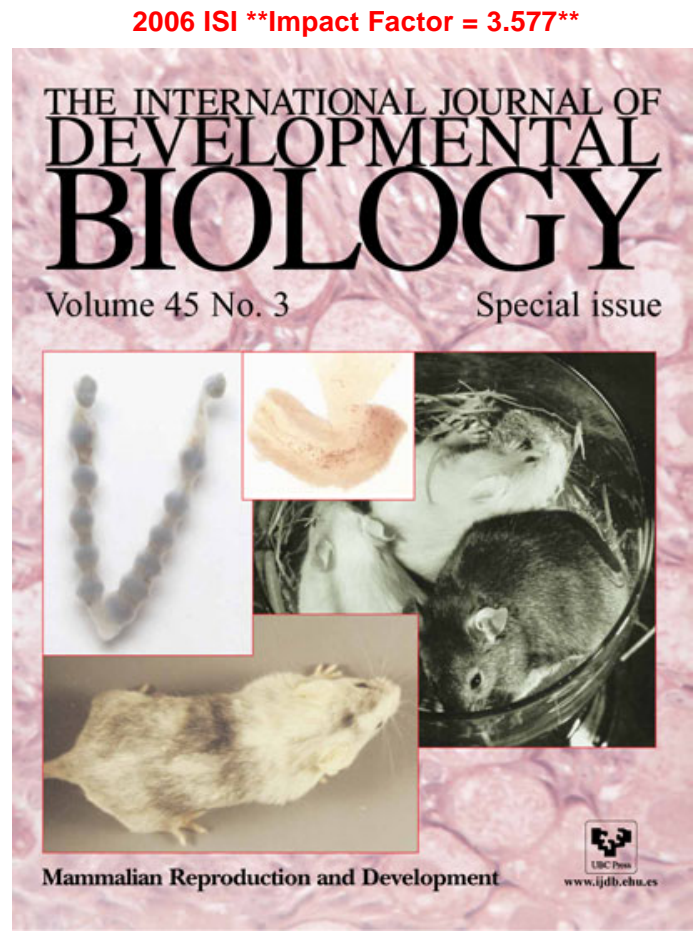

